\title{
Early Coronary Thrombosis without ST-Segment Elevation Following Repair of Acute Aortic Dissection
}

\author{
Davide Carino, MD*, Francesco Nicolini, MD, PhD, Giorgio Romano, MD, Matteo Ricci, MD, \\ Tiziano Gherli, MD
}

General and Specialist Surgery Department, Cardiac Surgery Unit, Parma General Hospital, Parma, Italy

\begin{abstract}
Acute coronary thrombosis after emergent surgery for acute Type $\boldsymbol{A}$ aortic dissection is a rare event that can remain undiagnosed in absence of typical electrocardiogram readings. We report a case of left anterior descending artery thrombosis without ST-segment elevation three days after surgical repair, which was successfully treated with angioplasty and stenting.

Copyright $\odot 2016$ Science International Corp.
\end{abstract}

\section{Key Words}

Acute aortic dissection - Coronary thrombosis • Acute coronary syndrome

\section{Introduction}

Acute coronary thrombosis after emergent surgery for acute aortic dissection (AAD) is a very rare but very serious event. In the absence of typical ST-segment elevation on the electrocardiogram (ECG), diagnosis can be challenging. Several different underlying mechanisms have been reported in the literature for coronary malperfusion following $A A D$, both before and after surgery. Selection of the best treatment for coronary thrombosis in the catheterization laboratory is also challenging, because it is necessary to carefully assess both the bleeding and thrombotic risk.

\section{Case Presentation}

A 67-year-old female with arterial hypertension presented to the emergency department with severe chest pain radiating to her back. The electrocardiogram was unremarkable and the troponin- $C$ was normal. Trans-thoracic echocardiograms revealed a dissection flap in the ascending aorta with normal left ventricular contractile function. A computed tomography (CT) scan confirmed the diagnosis of AAD with an intimal flap that extended from the ascending aorta to the iliac bifurcation. The dissection involved the innominate artery while the left carotid and left subclavian artery arose from the true lumen. No gross calcifications of the coronary vessels were detected. The patient underwent emergency surgery for AAD. After median sternotomy, femoro-atriocaval cardiopulmonary bypass was instituted and the patient was gradually cooled to $26^{\circ} \mathrm{C}$. After aortic cross-clamping, the dissected aorta was opened into the true lumen. Crystalloid cardioplegia was infused selectively into the coronary ostia. The ascending aorta was then replaced with a Gelweave (Vascutek Terumo, Tokyo, Japan) Dacron 30-mm straight prosthesis after fixing of the true and false lumens with resorcin-formaldehyde biological glue sandwiched between external and intra-luminal Teflon strips.

The first two postoperative days were unremarkable, with normal cardiac biomarkers. On the third

* Corresponding Author:

Davide Carino, MD

Cardiac Surgery Unit

Parma General Hospital, University of Bologna

Via Gramsci 14, 43126, Parma, Italy

Tel.: +39 0521 703270; Fax: +39 0521 702188; E-Mail: davidecarino88@gmail.com 
postoperative day, sudden hemodynamic instability appeared, with a growing requirement for inotropic drugs to maintain normal systemic pressure. No specific ECG changes were noted (Figure 1), but global left ventricle hypokinesia was noted on echocardiography without any segmental deficits. A sudden and severe rise in cardiac biomarker levels was also evident, forcing us to perform diagnostic coronary angiography. The catheterization was performed using a right brachial approach. The coronary angiogram revealed a thrombotic occlusion of the left anterior descending artery (LAD) (Figure 2). A 0.014" wire was advanced down the LAD and a $3.0 \times 15 \mathrm{~mm}$ Xience Pro (Abbott, Illinois, USA) was deployed, squeezing the thrombus peripherally (Figure 3 ). Double antiplatelet therapy with clopidogrel and acetylsalicylic acid was then administered. The remainder of the postoperative course was characterized by difficult weaning from the mechanical ventilation and a temporary tracheostomy. The patient was discharged on the 28th postoperative day in good clinical and hemodynamic condition.

\section{Discussion}

Several different underlying mechanisms have been reported for coronary malperfusion following AAD. The first is the so called "intimo-intimal intussusception": a circumferential intimal flap resulting from a tear near the sino-tubular junction that prolapses into the left ventricle during diastole, causing severe aortic regurgitation and occluding the coronary ostia [1]. Other mechanisms are the proximal extension of the false lumen toward the aortic root with involvement of the coronary ostia [2], which may also extend for several centimeters along the vessel [3], and acute

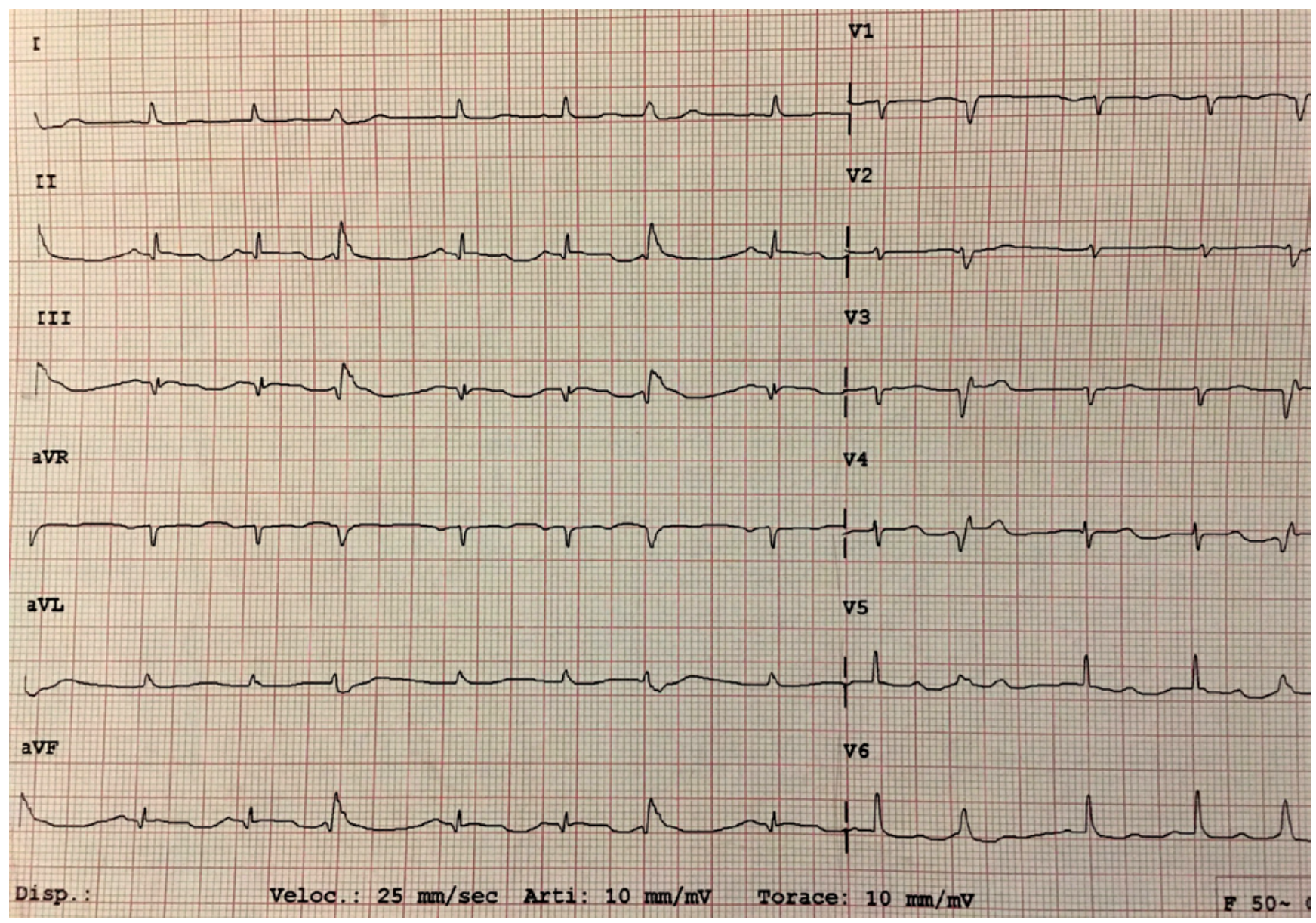

Figure 1. ECG showing non-specific repolarization changes but no ST-segment elevation. 


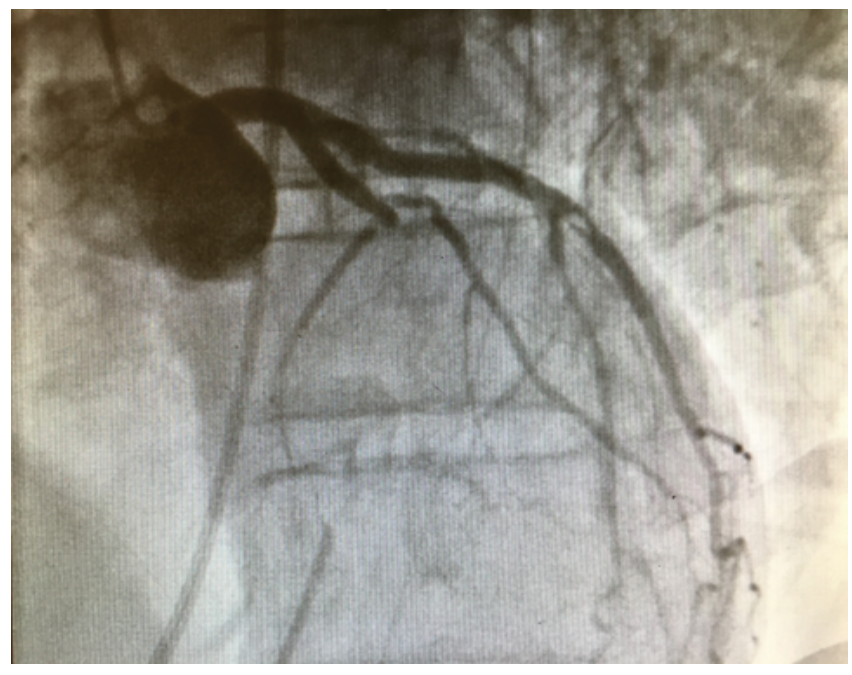

Figure 2. Coronary angiogram showing an acute occlusion of the left anterior descending artery.

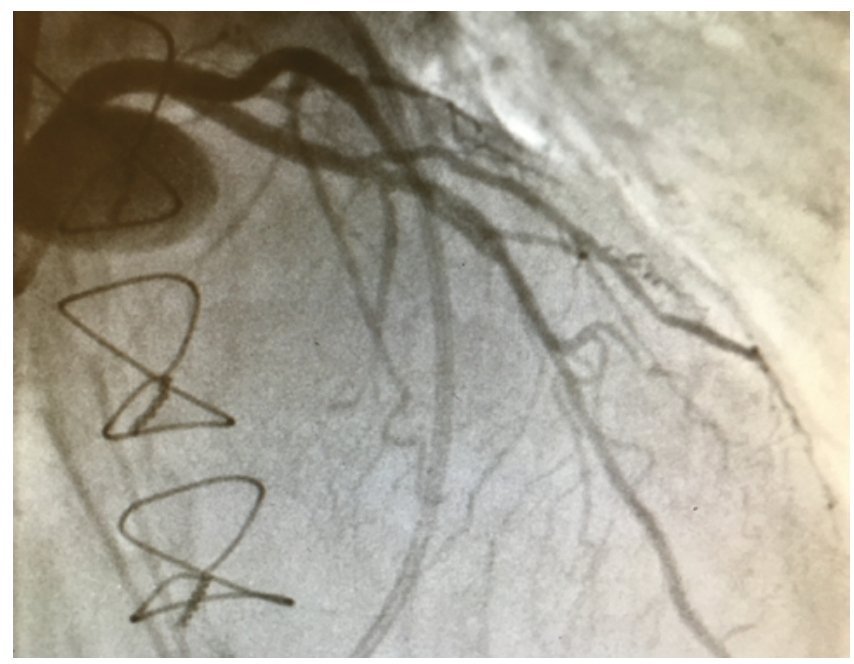

Figure 3. Demonstration of the satisfactory results of the percutaneous coronary intervention.

thrombosis of an atherosclerotic plaque immediately after replacement of the ascending aorta [4]. Embolization of glue fragments into the coronary artery has also been reported as a possible mechanism of coronary thrombosis after surgery for AAD [5]. Finally, late obstruction of the coronary ostia eight months after repair of aortic dissection has also been described, which was attributed to scarring caused by excessive application of formaldehyde glue [6].

In all cases previously reported, the clinical scenario was characterized by the presence of an STsegment elevation. In our case, the ECG did not show
ST-segment elevation, only non-specific repolarization changes. Moreover, the echocardiograph images showed global hypokinesia but no segmental contractile deficit was noted. The only evidence of acute coronary thrombosis was an inexplicable augmentation of the troponin and creatine kinase-MB levels on the third postoperative day. We carefully evaluated the risks and benefits of high-risk arterial catheterization in this patient who had just undergone surgery for AAD and did not have a fully thrombosed false lumen. Nevertheless, we were forced to perform a diagnostic angiography because of the otherwise inexplicable severe trend of the cardiac biomarkers and unstable hemodynamic status.

Another important aspect to consider is the high risk of bleeding related to the mandatory dual antiplatelet therapy after stent deployment in a patient who recently underwent an operation for AAD. A simple balloon angioplasty would have avoided the need for dual antiplatelet therapy.

In our case, the underlying mechanism was not clear, and could have been acute thrombosis of an atherosclerotic plaque or embolization of a fragment of glue into the coronary artery. The absence of gross calcifications of the coronary vessels at the CT scan indicate that the hypothesis of an acute thrombosis of an atherosclerotic plaque is unlikely, but our image of the thrombotic occlusion of the LAD is very different from the one reported by Hoschtitzky et al. [5] in which an embolization of a glue fragment was the underlying mechanism. We speculate that the occlusion of the LAD was caused by an acute thrombosis of an atherosclerotic plaque free of calcific component. A simple balloon angioplasty might not be effective, so we preferred to use a stent for the percutaneous coronary intervention, although this necessitated dual antiplatelet therapy and the consequent high hemorrhagic risk.

The presence of two concomitant conflicting diseases, such as AAD and acute coronary thrombosis, creates a very difficult scenario. The correct diagnosis presented a challenge for our heart team, especially because of the absence of ST-segment elevation, which was surprising considering the angiographic status. Selection of the best therapeutic option in the catheterization laboratory (stent or simple balloon angioplasty) was also difficult, as was the decision to 
administer antiplatelet therapy.

In conclusion, acute coronary thrombosis after repair of AAD is a very rare but very serious event. Regardless of the underlying mechanisms of the coronary thrombosis, a coronary angiography must be performed when an acute coronary syndrome is suspected after surgery for AAD.

\section{References}

1. Whitley W, Tanaka KA, Chen EP, Glas KE. Acute aortic dissection with intimal layer prolapse into the left ventricle. Anesth Analg. 2007;104:774-776. DOI: 10.1213/01. ane.0000256861.34798.63

2. Horszczaruk GJ, Roik MF, Kochman J, et al. Aortic dissection involving ostium of right coronary artery as the reason of myocardial infarction. Eur Heart J. 2006;27:518. DOI: 10.1093/eurheartj/ ehi525

3. Zegers ES, Gehlmann HR, Verheugt FW. Acute myocardial infarction due to an acute type A aortic dissection involving the left main coronary artery. Neth

\section{Conflict of Interest}

The authors have no conflicts of interest to declare.

\section{Comment on this Article or Ask a Question}

Heart J. 2007;15:263-264. DOI: 10.1007/ BF03085996

4. Marchetti M, Scacciatella $P$, Di Rosa E, Rinaldi M, Marra S. Coronary Thrombosis and Type A Aortic Dissection. J Card Surg. 2015;30:583-585. DOI: 10.1111/jocs.12577

5. Hoschtitzky JA, Crawford L, Brack M, Au J. Acute coronary syndrome following repair of aortic dissection. Eur J Cardiothorac Surg.2004;26:860-862. DOI: 10.1016/j. ejcts.2004.07.016

6. Tsukui $H$, Aomi S, Nishida $H$, Endo $M$, Koyanagi $\mathrm{H}$. Ostial stenosis of coronary arteries after complete replacement of aortic root using gelatinresorcinol- formaldehyde glue. Ann Thorac Surg. 2001;72:1733-1735. DOI: 10.1016/S00034975(01)02566-8

Cite this article as: Carino $D$, Nicolini F, Romano G, Ricci M, Gherli T. Early Coronary Thrombosis without STSegment Elevation Following Repair of Acute Aortic Dissection. AORTA (Stamford). 2016;4(4):138-141.DOI: http:// dx.doi.org/10.12945/j.aorta.2016.16.017 